\title{
An Entropy Approach to Tesla's Discovery of Wireless Power Transmission
}

\author{
Salama Abdelhady \\ College of Energy Engineering, Aswan University, Aswan, Egypt. \\ Email: salama_hady@cic-cairo.com
}

Received December $24^{\text {th }}, 2012$; revised January $26^{\text {th }}, 2013$; accepted February $9^{\text {th }}, 2013$

Copyright (C) 2013 Salama Abdelhady. This is an open access article distributed under the Creative Commons Attribution License, which permits unrestricted use, distribution, and reproduction in any medium, provided the original work is properly cited.

\begin{abstract}
Following an entropy approach, the electric current can be postulated as a flow of electromagnetic waves that has an electric potential. Such postulate is verified in this article by results of experimental measurement then it is applied to review Tesla's discovery of wireless power transmission. Tesla discovered the wireless transmission of electric power during his operation in an entirely new domain of physics based on abrupt discharging of electrostatic charges. He realized that electrons were not responsible for such discovered phenomena. So, he called the new energy as "Radiant Electricity". He didn't identify that the true nature of electric current was a flow of electromagnetic waves that can be normally radiated through air. So, he proposed the term "Aether gas" to describe the discovered transfer of the electric charges by radiation from his designed tower, though air, to specified receivers. Following an entropy approach, the nature of the discovered radiated energy can be identified as electromagnetic waves that have an electric potential. So, the followed approach succeeds in eliminating the confusing observations of Tesla's measurements in his famous experiment and the confusions found in modern literature that deals with such energy as an "Ambiguous Radiant Energy".
\end{abstract}

Keywords: Entropy; Tesla’s Discovery; Radiant Energy; Electromagnetic Waves

\section{Introduction}

Nikola Tesla is seen by mostly scientists as the "Father of Wireless Power Transmission" or the first person to conceive the idea of wireless power transmission and successfully demonstrated the transmission of electricity without wires as early as 1891 [1]. In 1893, Tesla won to illuminate the World Columbian Exposition in Chicago, where he demonstrated the success of illuminating the vacuum tubes bulbs without using wires. Tesla constructed a tower, Wardebclyffe tower, for wireless power transmission of electrical power rather than telegraphy [2]. In his experiment, Tesla gave rise to the electrostatic charge to a maximum, and discharged it much more quickly through a tentative circuit that resembled an electric-pulse jet [3]. So, Tesla operated in a domain of physics basedon abrupt discharge of electrostatic charge through air which he referred to as "dynamic electrostatic" [4]. This process simulates the discharge of clouds to earth where electricity can be attracted and earthed through specially designed metallic rods [5]. Tesla and his colleagues found that traditional definitions of electricity as a flow of electrons through a conductor (like a wire) cannot explain such phenomena. However, Tesla and others believed that the electrical forces were actually streams of Aether gas, which had been fixated in matter [6]. Tesla also approached the definition of electric current as "radiant energy" [7]. Similarly, Hertz identified also this transferred power as electrostatic inductions or electrified shock waves that resemble true electromagnetic waves [8]. Finally, Tesla described the travelled charges as light-like rays but he supposed its nature is different from the electromagnetic waves of Maxwell [9].

According to an entropy-approach and the similarity characterizing the heat flow and the flow of electric charges, such "Radiant Energy" can be postulated as a flow of electromagnetic waves that have an electric potential [10]. Main concepts and experimental results that verify the truth of such postulate will be reviewed in the next section. Then it will be reviewed the significance of Tesla's interpretation of his discovery of the wirelessly transferred energy as "Radiant Energy" or Aether gas in his new domain of physics according to the followed entropy approach. Finally, it will be shown how the introduced entropy and the postulated definition of electric 
current as a flow of ionized electromagnetic waves give the convenient answers for most of the published Tesla's inquiries in his experiments.

\section{Definition of Electricity as Electromagnetic Waves}

Starting from the similarity and analogy of laws governing the flow of heat and electricity, it was possible to postulate that such flows have the same nature [10]. In other words, the nature of electric current is postulated as the heat radiation in the form of electromagnetic waves. However, heat radiation is a form of electromagnetic waves that have a thermal potential. Similarly, the flow of electric charges can be postulated as a form of electromagnetic waves that have an electric potential [11]. Accordingly, the photoelectric effect is interpreted as a process of ionizing the incident light during its reflection on the metal plate of a photocell [12]. This plausible explanation is verified according to the measurement results of the potential that stop the flow of photocurrents in photocells. Such potential is found as a function of the incident light-frequency and the type of the metal of the reflecting plates. The measurement results prove that the reflected light in the photocells gains after its reflection a specific potential that depends on its frequency and on the electrode potential of the reflecting metal plate. So, the flow of current from the photocells is a flow of ionized photons that have a potential related to the frequency of the incident light and it is not a flow of electrons of zero potential that might be emitted from the metal plate by impact of massless light-waves [13]. The plots that show the dependence of the measured stopping potential of photocurrent on the frequency of the incident light on two metals. Figure 1 has the same slope for thetwo metals and it is equal to the well known Planck's constant " $h=6.626 \times 10^{-34} \mathrm{~J} \cdot \mathrm{s}$ ". The results are represented by the following relation between the frequency of the incident light " $v_{\text {incident }}$ " and the potential " $V$ " of the reflected waves or photons after ionization by the metal

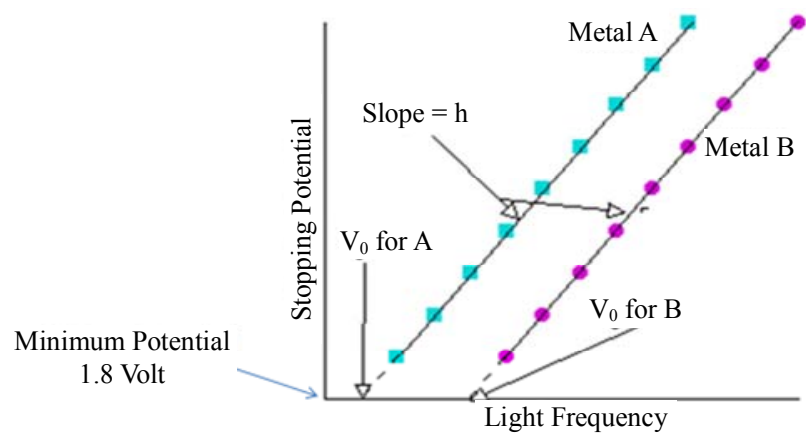

Figure 1. Plot of the measured stopping potentials obtained for several light frequencies in photocells for two different metals [14]. plate that defines the known physical constants $h$ and $e$ [14]:

$$
h * v_{\text {incident }}=e * V
$$

The symbol " $e$ " in Equation (1) indicates the charge of the reflected photons $\left(e=1.6 * 10^{-19}\right.$ Joule/Volt $)$. As seen in Figure 1, the frequency of the light that can be converted into an electric current and gains after reflection a potential should be higher than a minimum or "threshold frequency $v_{o}$ " [14]. The value of such threshold frequency may be found directly from measurement of the minimum potential that stops the photocurrent. According to Figure 1, this value is found as " $V_{\text {minimum }} \cong 1.8$ Volts ." Such value depends on the electrode potential of the metal plate and the values of the constants in Equation (1) [14].

Similar proof can be concluded according to the measurement results of light emitting diodes [15] (Figure 2). Such results prove also a nature of the electric flux as ionized electromagnetic waves. The process in the diode can be interpreted as deionizing the flowing electric current through the diode by the built-in potential of the LED's p-n junction. So, the incident electric current, as ionized photons that have a specific potential, is converted into light waves of zero potential. Figure 2 shows the measured frequency of emitted light from a diode as a function of the applied potential for different types of diodes. Increasing the potential of the incident current on such LEDs lead to increasing the frequency of the emitted light too according to an equation similar to the photocell's Equation (1) $[15,16]$ :

$$
e * E_{0}=h *\left(\frac{c}{\lambda_{\text {emitted }}}\right)=h * v_{\text {emitted }}
$$

In Equation (2), “c $c=300 \times 10^{6} \mathrm{~m} / \mathrm{s}$ " is the speed of light and " $\lambda$ " is the wavelength of the emitted light in meters. The measured slope of the curve in Figure 2 determines the same value of Planck's constant as found from the measured slope in Figure 1 for photocells.

According to the measurement results in Figure 2, for each diode there is a minimum potential of the flowing current, called threshold potential " $V_{\text {thresh }}$ or $V_{0}$ " that led to emit light from such diode [15]. Figures 2 and 3 clearly show the correspondence between the processes in the LEDs and photocells. The same slopes of both lines and the correspondence between the threshold frequency in Figure 2 and the threshold potential in Figure 3 indicate that the processes in the diodes and the photocells are process of ionizing and de-ionizing the light waves or photons. So, photocells and diodes that have the same frequency bands are working with the same potential drop or gain as they are operating on the same waves but proceeding in opposite directions. So, the postulated definition of the flow of electric charges as a flow of 


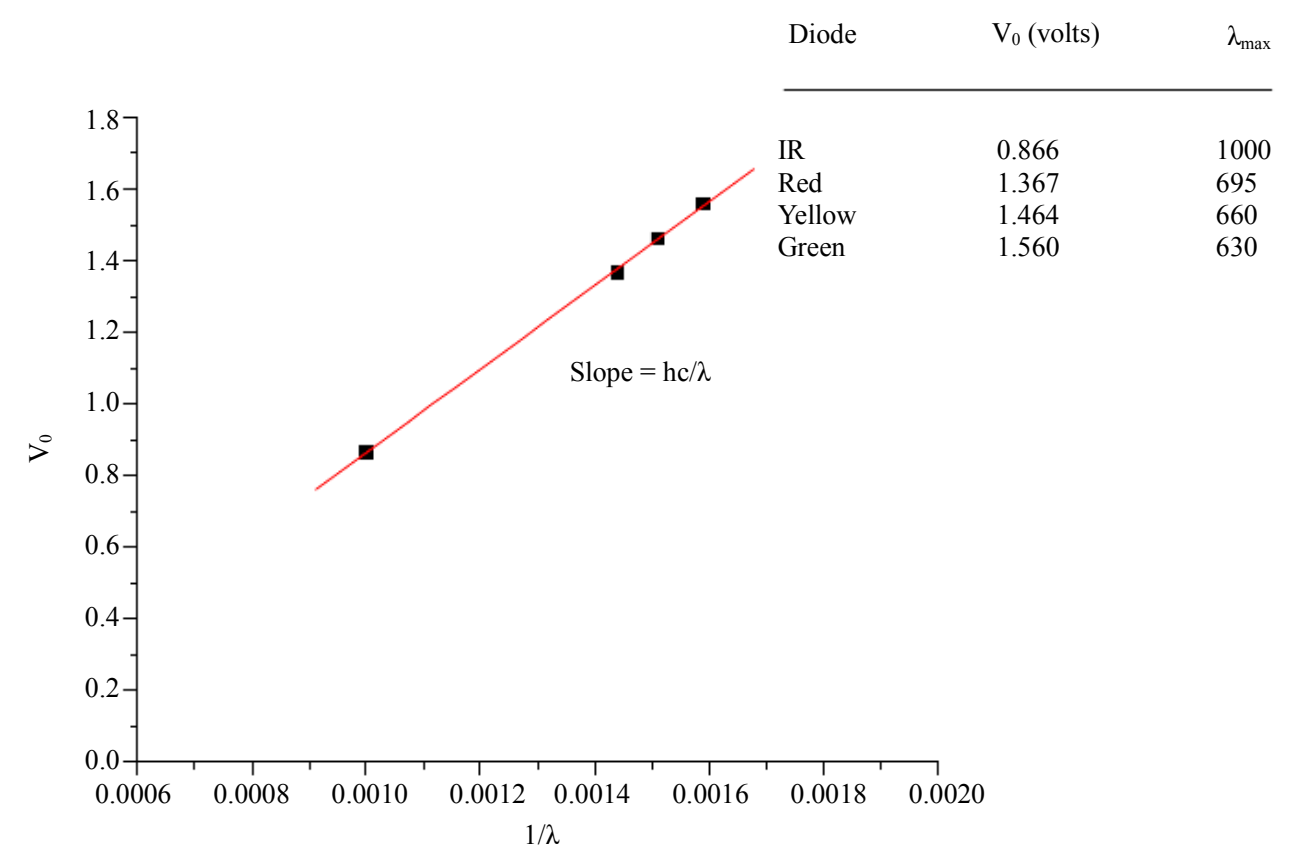

Figure 2. Determination of Planck's constant using LED’s [15].

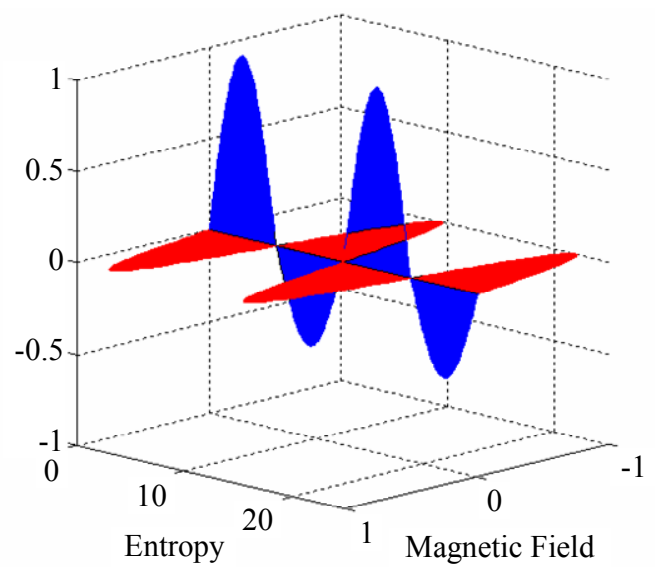

Figure 3. Electromagnetic wave that represent flow of positive charge of potential $E_{0}$.

ionized electromagnetic waves finds also a plausible explanation for the processes in the diodes and the photocells. Equations (1) and (2) can be simplified by one proportionality equation as follows $[16,17]$ :

$$
\lambda_{\text {incident or emitted }} \propto \frac{1}{V_{\text {photocell or diode }}}
$$

According to such results, the potential of the electric current as flow of electromagnetic waves depends on the frequency or the energy of such waves. So, the following equation may express the output current from a photocell or the incident current in a diode [1]:

$$
E=E_{0}+E_{1} \cos (\omega t)
$$

where $E$ is the potential of the oscillating wave around and $E_{0}$ is the threshold potential. Such equation can be considered as a solution of the Maxwell's wave equations that belongs to the electric component of the electromagnetic wave [10]:

$$
\begin{aligned}
& \left(\nabla^{2}-\frac{1}{c^{2}} \frac{\partial^{2}}{t^{2}}\right) E=0 \\
& \left(\nabla^{2}-\frac{1}{c^{2}} \frac{\partial^{2}}{t^{2}}\right) B=0
\end{aligned}
$$

The arrow of time is found as a property of entropy alone [11]. Accordingly; time in Maxwell Equations (5) and (6) may be replaced by entropy. Such transformation leads to modified solutions of Maxwell equations that may express the flow of electric charges into potentialentropy coordinates as follows:

$$
E=E_{0}+E_{1} \cos (\omega s)
$$

Such equation can be represented in graphically in the E-s coordinates as shown in Figure 3 [11].

Figure 3 represents the flow of positive charges, according to Equation (6), as flow of electromagnetic waves of positive potential [11]. The advantage of such coordinate system is its representation of the energy propagation into a system by the intercepted areas in a diagram. As an example, the heat flow is represented in the temperature-entropy diagram by the by the following integral [11]:

$$
Q_{\text {thermal }}=\int T \mathrm{~d} S
$$

By similarity between the electric charge and heat 
flow as both are forms of electromagnetic waves and are driven by their associated potentials $E$ or $T$, the flow of electric charge can be expressed also by areas in an E-s diagram according to the following equation [11]:

$$
Q_{\text {elect }}=\int \bar{E} \mathrm{~d} S_{\text {elect }}
$$

According to Figure 3, the area representing the integral in Equation (9) has a net positive sign or a charge of positive potential. So, the flow of electricity is a flow of electromagnetic waves that have a definite potential or propagation of entropy by virtue of a definite electric potential. According to the general thermodynamic principles, temperature as a potential of heat represents the quality of the propagated heat while entropy propagation measures a quantitative influence of the energy flow on the system. Such principles may be applied to the flow of electric charge where the electric potential represents the quality of the charge while entropy measures the quantitative influence of the flow of electricity on the system.

\section{An Entropy Analysis of Tesla's Conclusions}

According to the previous entropy approach, the accumulation of electric charges that possess electrical potentials on the top surface of the Tesla's tower [3], leads to summation of potentials of these charges to extremely high values, as measured by Tesla [3], according to the following equation:

$$
E_{\text {tower }}=\sum_{i=1}^{N} E_{0 i}
$$

$E_{0 i}$ is the potential of each charge which was defined as the thresholds' potential $\left(V_{i}\right), N$ is the number of accumulated charges on the top of the tower and $E_{\text {tower }}$ is the potential of the accumulated charges on the top of the tower. When such high potential energy is discharged as electromagnetic waves, it will produce the measured shock wave in a similar fashion as the discharged of clouds [5]. According to Equation (9), the rate of flow of entropy from the tower to the receiver during the discharge process can be expressed as follows:

$$
\dot{S}_{\text {electrical }}=\frac{\dot{Q}_{\text {electrical }}}{E}
$$

So, the energy transmitted from the tower to the receiver is a flow of entropy by force of the tower's potential and can be estimated according to the following equation:

$$
\dot{Q}_{\text {electrical }}=E \dot{S}_{\text {electrical }}
$$

However, the loss of the transmitted electric energy from the tower to the end receivers, $\delta\left(E \dot{S}_{\text {electrical }}\right)$, is dissipated to the air in the form of thermal energy. The dissipated energycan be estimated by applying an energy equation that embraces the flow of electric and thermal energy [11]:

$$
\delta\left(T \dot{S}_{\text {thermal }}\right)+\delta\left(E \dot{S}_{\text {electrical }}\right)=0
$$

So, the lost electric charge will be gained by air as thermal energy $\delta\left(T \dot{S}_{\text {thermal }}\right)$ that will increase the air temperature. If the attained temperature is high enough, it may ignite atmospheric nitrogen in presence of atmospheric oxygen according to the following chemical equations [18]:

$$
\begin{aligned}
& \mathrm{N}_{2}+\mathrm{O}_{2} \rightarrow 2 \mathrm{NO} \\
& \mathrm{N}_{2}+2 \mathrm{O}_{2} \rightarrow 2 \mathrm{NO}_{2}
\end{aligned}
$$

Similar reactions are found in thunder storms during discharge of clouds [8]. The accumulation of charge on clouds leads to the rise of its potential in a similar process as charging of Tesla's tower and its discharge in a short time leads to burning of forests. Numerical examples and measurements of such energies are available in literature and are applied in the design of grounding networks [5]. So, the change of colors during Tesla experiment is due to the relative activation of nitrogen burning according to Equation (14a) or (14b) [18]. In other words, the seen colors of the radiated energy (Figure 4) were not belonging to Aether gas, as was claimed [6], but it actually belonged to burning of nitrogen in the surrounding air.

Such conclusions violate the definition of electricity as flow of electrons that requires conducting wires and sustains its postulated definition as a flow of electromagnetic waves [11]. In other words, Tesla's radiated energy should be defined as ionized electromagnetic waves that have specific potential and it is not a stream of gas. It is actually a flow of entropy by the influence of the sum of the potentials of the accumulated charges.

According to the previously discussed measurement results of photocells and diodes, the wavelengths of the

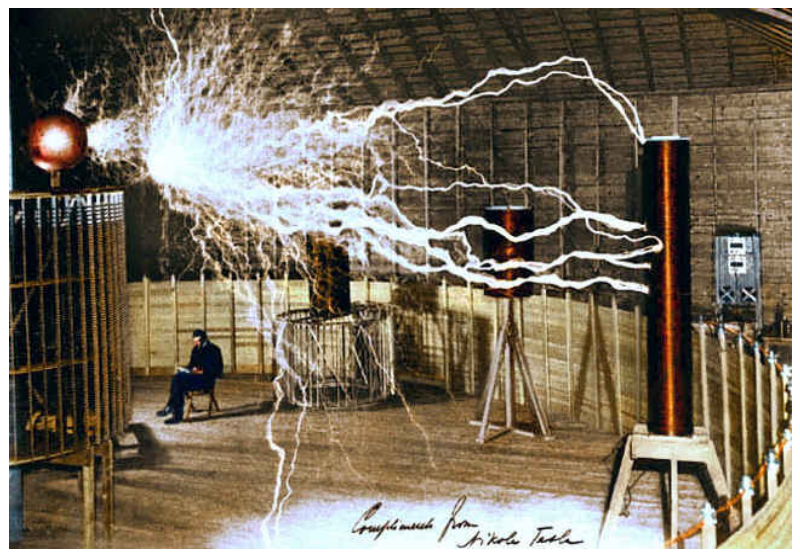

Figure 4. One of Tesla's experiments on radiated energy [1]. 
ionized electromagnetic waves that define a flow of electric charges are in the range of $650-1500 \mu \mathrm{m}$ [19]. This range belongs mostly to the invisible range of electromagnetic waves. Accordingly, the followed entropy approach defines the transferred power as electromagnetic waves whose spectrum does not belong to the lightspectrum. So, such transferred power is really as Tesla presumed; "Dark Radiation" which should not be interpreted as some authors claim a Secret energy or Scalar Energy. Tesla's experiment discovered the true nature of the flow of electric charges as a flow of ionized electromagnetic waves.

It is an interesting remark of Tesla that he had never referred to "space-time" directly. He considered time scale or dimension as a mere man-made "measure" of the rate at which events may occur such as a distance travelled in a certain period of time, for a frame of reference. He tried to find another frame of reference that ties such the space to the system's energy but he didn't find a logical substitute [20]. In the previously explained entropy approach, time in the previous Maxwell's wave equations is replaced by entropy which measures the influence of energy propagation since time is found as a property of entropy alone [12]. Hence, such replacement may represent the logical substitution that Tesla failed to find [20]. According to such substitution, the radiant energy represent a flow of entropy by the influence of a high accumulated potential, due to accumulation of electric charges, and it is not flow of Aether gas or scalar energy according to Tesla's confusions.

\section{Conclusion}

According to an entropy approach and a review of available experimental results, the flow of electricity is realized as flow of electromagnetic waves that have an electric potential. So, Tesla's discovery of "Radiant energy" should be also interpreted as normal radiation of electromagnetic waves that have an electric potential. Replacing the time coordinate in Maxwell's wave-equations by entropy, the discovered "Radiant Energy" of Tesla is also found as a flow of entropy by the influence of its electric potential. Such radiant energy is also found as a dark energy or electromagnetic waves whose wave lengths are not in the visible spectrum.

\section{REFERENCES}

[1] K. Adachi, "Radiant Energy, Unraveling Tesla Greatest Secret," 2001. http://www.gnosticliberationfront.com/radiant_energy.htm

[2] R. Biswa, "Feasibility of Wireless Power Transmission," 2012.

http://www.academia.edu/1561057/Feasibility_of_Wirele
ss_Power_Transmission

[3] D. Mitchell, "Nikola Tesla's Investigation of High Frequency Phenomena and Radio Communication," 1972. http://www.mentallandscape.com/tesla2.htm

[4] J. Aceret, "Radiant Power Unraveling Tesla's Greatest Technique," 2012.

http://www.selfgrowth.com/articles/radiant-power-unrave ling-tesla-s-greatest-technique-0

[5] A. Gosline, "Thunderbolts from Space," New Scientist, Vol. 186, No. 2498, 2005, pp. 30-34.

[6] A. Waser, "Nikola Tesla's Wireless Systems," 2000. http://www.andre-waser.ch/Publications/NikolaTeslasWir elessSystems.pdf

[7] N. Tesla, "The Effect of Static on Wireless Transmission," Electrical Experimenter, 1919, pp. 627-658.

[8] J. T. Ratzlaff, "Dr. Nikola Tesla: Selected Patent Wrappers," Tesla Book Company, 1980, p. 150.

[9] S. K. Singm, T. S. Hasarmani and R. M. Holmukhe, "Wireless Transmission of Electrical Power Overview of Recent Research \& Development," International Journal of Computer and Electrical Engineering, Vol. 4, No. 2, 2012, pp. 207-211.

[10] S. Abdelhady, "An Entropy-Approach to the Duality Property," Journal of Electromagnetic Analysis \& Applications, Vol. 3, 2011, pp. 220-227.

[11] S. Abdelhady, "A Fundamental Equation of Thermodynamics that Embraces Electrical and Magnetic Potentials," Journal of Electromagnetic Analysis \& Applications, Vol. 2, 2010, pp. 162-166.

[12] S. Abdelhady, "Comments on Einstein's Explanation of Electrons, Photons, and the Photo-Electric Effect," $\mathrm{Ap}$ plied Physics Research, Vol. 3, No. 2, 2011, pp. 230-240.

[13] S. Abdelhady, "A Thermodynamic Analysis of Energy Flow in Optical Fiber Communication Systems," Applied Physics Research, Vol. 4, No. 3, 2012, pp. 22-29. doi:10.5539/apr.v4n3p22

[14] M. Hackworth, "Measuring Planck's Constant," 2000. http://www2.cose.isu.edu/ hackmart/planck's.PDF

[15] S. Ducharme, "Measuring Planck's Constant with LEDs," 1999. http://physics.unl.edu/directory/ducharme/ducharme.html

[16] S. Hüfner, "Photoelectron Spectroscopy Principles and Applications," Springer, Berlin, 2003. doi:10.1007/978-3-662-09280-4

[17] S. M. Sze, "Physics of Semiconductor Devices," John Wiley \& Sons, New York, 1981, pp. 74-81.

[18] Allen, et al., "Impact of Lightning NO Emissions on North American Photochemistry as Determined Using the Global Modeling Initiative (GMI) Model," Journal of Geophysical Research, Vol. 115, No. D22, 2010.

[19] W. Sinnema, "Electronic Transmission Technology," Electronic Transmission Technology Prentice Hall, 1979.

[20] M. Cheney, “Tesla-Man out of Time," Prentice Hall, 1981. 